\title{
Guided bone regeneration with Pericardium membranes
}

\author{
Dr. Swati Gupta ${ }^{1}$, Dr. Richa Gupta ${ }^{2}$ \\ ${ }^{\prime}$ (MDS Periodontology, Chandigarh, India) \\ ${ }^{2}$ (MD Anatomy, PGIMER, India)
}

\begin{abstract}
There is a plethora of evidence suggesting the role of barrier membranes in bone augmentation at sites of inadequate bone volume and height. Amongst the resorbable membranes, native collagen membranes are the standard treatment for guided regeneration procedures. However, native collagen resorbs rapidly whereas cross linking delays it. Pericardium membranes are multilayered, resulting in prolonged barrier function with the properties of native collagen intact. These membranes have proved their utility in the field of vascular, cardiac, thoracic surgery because of their mechanical and clinical handling properties. However, there is very little evidence of bone augmentation with pericardium membranes in the field of dentistry, particularly Implantology. The purpose of this review is to discuss the potential applications of these membranes in guided bone regeneration.
\end{abstract}

Keywords: pericardium, barrier membranes, guided bone regeneration, bovine membranes

\section{INTRODUCTION}

Periodontal therapy has almost always focused on the arrest of the disease progress and maintenance of the remaining periodontal support. Treatment to restore periodontal health and achieve restitution of attachment apparatus has varied based on the etiology and encompasses procedures such as root planing, soft tissue curettage and various types of flap procedures, often in combination with the placement of bone grafts or bone substitutes into the defects. Most procedures have merely brought about tissue repair, which signifies "replacement of the lost apparatus with scar tissue which does not completely restore the architecture or function of the part replaced". Regenerative procedures currently in use in periodontal therapy have not been able to achieve regeneration in the true sense. There have been reports of gaining new attachment by the formation of new cementum.[1] However; bone formation is largely insubstantial or delayed.

Melchers hypothesis of selective cell repopulation contributed to the understanding of the mechanics of wound healing. This concept was used further by series of experiments and formed the basis of the biologic principle of Guided tissue regeneration. Guided tissue regeneration (GTR) "is used to define procedures wherein regeneration of lost periodontal structures (cementum, periodontal ligament, and alveolar bone) is sought via selective cell and tissue repopulation of the periodontal wound". Periodontal literature is replete with the research work related to tissue and bone regeneration achieved with GTR. This concept further evolved to a more evolved compartmentalized principle of guided bone regeneration (GBR). The biological basis for guided bone regeneration involves fulfillment of bone growth requirement: establishing stable immobile base, allow for release of growth factors, and finally, preserving the blood supply to the area of defects. The guided bone regeneration promotes bone formation by protection against invasion of competing, non osteogenic tissues with the use of barrier membranes. There are three generation of membranes which includes resorbable membranes like collagen and polylactide-co- glycolide, non resorbable membranes like ePTFE and Gore tex and few other membranes under investigation which includes membranes like alloderm, gelfoam, cargile and pericardium membranes. The purpose of this review is to throw light on the potential application of pericardium membranes in GBR.

The pericardium is a fibroserous sac surrounding the mammalian heart. It has long been used in cardiac repair for reconstruction, valve repair and pericardial closure.[2, 3] Pericardial tissue has exceptional handling characteristics and uniform suture retention. In addition, it is nonthrombogenic and naturally resists infection. Xenogenic pericardium is commonly derived from bovine, porcine and, less frequently, equine sources. Tissues from these sources are available in large patches, allowing custom configuration to a variety of cardiovascular applications. It is largely comprised of collagen fibers and has elastic properties allowing conformity to complex anatomy. [4]

Bovine pericardium found its application initially as patches for arterial closure during vascular and cardiac surgery. The native structure of bovine pericardium has three layers: 1) the serosa, the inner thin layer consisting of mesothelial cells; 2) the fibrosa, the thicker layer formed by diversely oriented, wavy bundles of collagen and elastin; and 3) the epipericardial connective tissue layer, the outer layer that is partly continuous with the pericardiosternal ligaments. [5] BP is widely used in cardiac and thoracic surgery, as bioprosthetic valve leaflets, for repair of intracardiac defects, for repair of diaphragmatic defects, vascular surgery, including general surgery, urologic surgery and ophthalmology. The various beneficial properties promoting the use of 
bovine pericardium in tissue regeneration is because of its acellularity, reliable consistency, ability to be manufactured and processed to a consistent nominal $0.5 \mathrm{~mm}$ thickness, providing dependable suture retention and ideal operative handling characteristics. These properties provide a natural microenviroment for host cell migration and proliferation, accelerating endothelialization and tissue regeneration.[6] To maintain ECM content and structure, and to strengthen the biological tissue without causing calcification in vivo, approaches have included treating the glutaraldehyde-processed pericardium with glutamic acid, modifying the decellularization procedure [7], and nanocoating pericardial grafts with titanium to prevent immune reactions and thus calcification. [8] One of the trials suggested that cellular tissue-engineered bovine pericardium coated with poly(L-lactic acid)-co-poly(e-caprolactone) (PLACL) / blend of PLACL and gelatin nanofibrous scaffolds, along with human bone marrow-derived MSCs differentiated into endothelial cells, might improve the scaffolds' functionality for tissue engineering.[9] Puros ${ }^{\circledR}$ Pericardium, Copios pericardium manufactured by Zimmer dental are designed for guided tissue regeneration and guided bone regeneration procedures. It retains the natural collagen matrix and mechanical properties of native pericardium due to the proprietary Tutoplast ${ }^{\circledR}$ Process. It is characterized by its multidirectional strength, quick hydration, five-year shelf life and room temperature storage. These have a resorption profile of 4 to 6 months. The timeframe for remodeling depends upon the site; patient age, health and metabolic and nutritional status; biomechanical load on the graft. Three convenient sizes can be cut to shape for specific procedures. An experimental study was performed to choose the best procedure on preparation of acellular bovine pericardium (ABP) guided bone regeneration (GBR) material. BP was decellularized with $0.25 \%$ Trypsin $+0.5 \%$ Triton X-100. The acellular bovine pericardiums (ABPs) were treated with phosphate-buffered saline (PBS) (group A), 95\% glycerol (group B), EDAC (group C), and 1-ethyl-3(3-diaminopropyol)-carbodiimide (EDAC) and 95\% glycerol (group D) respectively. It was concluded that ABP treated with EDAC can be replaced by the surrounding tissues and has good biocompatibility.[10] A study has provided evidence of tissue regeneration within a porous bovine pericardium through a process involving cell recruitment and tissue-specific differentiation.[11] Owing to its properties; bovine pericardium has been accepted in the field of dentistry and finds its usage in the following areas:

1. Alveolar ridge augmentation: Bovine pericardium in conjunction of xenografts has been used in the augmentation of alveolar ridge defects in a study by Steigmann. 8 systemically healthy patients' ridge defects were augmented with bovine xenograft and a collagen pericardium membrane. Horizontal (width) hard tissue measurements were taken the day of ridge augmentation surgery, or implant placement and augmentation (baseline), and at the 6-month (reentry or uncovering) surgery. The change in ridge width varied from a loss of $0.2 \mathrm{~mm}$ to a gain of $7.8 \mathrm{~mm}$, measured clinically with a mean value of 3.04 and a median of $2.8 \mathrm{~mm}$ from baseline. The results suggested that pericardium collagen membrane might be a suitable component for augmentation of localized alveolar ridge defects.[12] Similar results were reported in a recent multicenter study.[13]

2. Guided bone regeneration: The microarchitecture, mechanical properties, cytotoxicity and cell chemotaxis properties of the acellular BP was evaluated in vitro, and the in-vivo efficacy of the acellular BP was also investigated in a rabbit mandibular model. The results suggested that acellular BP membrane possessed an interconnected fibrous structure. Glutaraldehyde (GA) treatment was efficient for enhancement of the mechanical properties of the acellular BP bur and resulted in negligible cytotoxicity. After $16 \mathrm{wk}$, standardized osseous defects created in the rabbit mandible, and covered with acellular BP, were associated with an enhanced deposition of mineralized tissue when compared with defects left to spontaneous healing.GA-treated acellular $\mathrm{BP}$ was found to be promising as a barrier membrane for GBR for further in-vivo and clinical studies.[14] Mild inflammatory response has been observed with the use of Glutaraldehyde (GA) than the no membrane group in bone defects prepared in dog mandible, however the healing after 4 weeks was found to be comparable. [15] Bovine pericardium promotes new bone formation by inhibiting new connective tissue invasion.[16] An animal trial was conducted to compare the efficacy of bovine pericardium in bone formation in critical sized defects as compared to collagen membrane. Histologically, the results showed that surface of bone defects was even, consistent with adjacent normal bone in EDAC-crosslinked ABP group, while that of bone defects was of no evenness in CM group in the 16th and the 24th weeks. There were no significant differences in new bone percentage of bone defects between 2 groups in the 4th and the 8th weeks $(\mathrm{P}>0.05)$. In the 16th week new bone percentage of bone defects was $81.99 \%+/-3.92 \%$ in EDAC-crosslinked ABP group and $76.35 \%+/-4.29 \%$ in $\mathrm{CM}$ group, showing significant difference $(\mathrm{P}<0.05)$. The average percentage of absorption in EDACcrosslinked ABP group was $16.57 \%, 27.94 \%, 65.61$ and $85.72 \%$ in the 4th, 8th, 16th and 24th weeks respectively, while that in CM group was more than $50 \%$ in the 4th week and completely degraded at the end of 8 weeks.[17] The clinical and histologic healing of deep intrabony defects treated with guided tissue regeneration (GTR) was assessed with a bovine pericardium and implantation of granular bovine bone biomaterial. Healing was uneventful in all patients. Significant clinical improvements were observed at 1 and 3 years postoperatively $(\mathrm{P}<0.01$; probing depth averaged $4.4-1.6$ and $4.7-1.4 \mathrm{~mm}$ and clinical attachment level gain was $3.9-1.4$ and $3.5-1.3 \mathrm{~mm}$, respectively). The histologic evaluation revealed formation of new cellular 
cementum and new periodontal ligament in four of the five cases. In general, the xenograft particles seemed to be mostly embedded in connective tissue without any evidence of new bone formation.[18]

3. Root coverage: Novel bovine pericardium based, non-cross linked collagen matrix has been tried for root coverage in 62 gingival recessions of Miller class I or II. With a baseline value of $1.5 \pm 0.9 \mathrm{~mm}$ an improvement of $2.4 \pm 0.8 \mathrm{~mm}$ after six month could be observed. 40 out of 62 recessions were considered a thin biotype at baseline. After 6 months all 62 sites were assessed thick. Also changes in clinical attachment levels were significant.[19]

4. Treatment of dehiscence defects: A study investigated the effect of a membrane via Sandwich bone augmentation for the regeneration of buccal implant dehiscence defects. Twenty-six healthy patients, each with a single defect, were randomly assigned into two groups. Both groups received an inner and outer layer of mineralized human cancellous and cortical particulate allograft. In the test group, a bovine pericardium membrane covered the bone grafts, while no membrane was placed in the control group. Cone beam computed tomography $(\mathrm{CBCT})$ scans were taken before and immediately after implant placement and at 6 months postsurgery. Clinical re-entry measurements showed significant buccal bone gain in the test group compared with the control group $(P<0.05)$. Cone beam computed tomography analysis demonstrated significant buccal bone gain of $1.22 \mathrm{~mm}$ in the test group. Radiographic vertical bone loss at 1 -year post-surgery showed no significant differences between the groups. These results led to the conclusion that addition of a barrier membrane prevented significant horizontal buccal bone resorption as space was maintained more effectively when compared with sites treated without a membrane.[20]

\section{Porcine Pericardium}

Pericardium from bovine and porcine sources present with slight differences. Pericardium from bovine sources has higher collagen content than that derived from porcine origins and they do not exhibit a significant difference in the degree of calcification under varying glutaraldehyde treatments. Valves fabricated from bovine pericardium have shown less obstruction than valves made from porcine pericardial tissue, although both valves show similar hemodynamic results.[21] In the field of cardiac surgery, Porcine pericardium represents a plausible scaffold option for the regeneration of the mitral leaflets in vitro or in vivo, respectively. Decellularized pericardium has been shown to be an optimum material for cell repopulation, delivering the necessary biological and biomechanical cues to seeded or migrating cells.[22] A pilot study was conducted to compare the in vitro and in vivo properties of a novel native collagen membrane extracted from porcine pericardium. Three different native collagen membranes (Remotis ${ }^{\circledR}$ Membrane [RM] porcine pericardium, Thommen Medical, Waldenburg, Switzerland; Bio Gide ${ }^{\circledR}$ [BG], porcine collagen types I and III, Geistlich Biomaterials, Wolhusen, Switzerland and Tutodent ${ }^{\circledR}$ [TD], bovine pericardium,Zimmer Dental, Freiburg, Germany] were incubated with $1 * 104$ SaOS-2 osteoblast-like cells. After 2 hours, 3 and 7 days, surface cell proliferation and the morphological structure were evaluated using a scanning electron microscope. The histological examination of the biodegradation after application was evaluauted in a dog model. In vitro RM and TD showed considerable cell proliferation, which was significantly superior to that observed with $B G(p<0.05$, ANOVA). An interconnective pore system was identifiable for RM, while BG displayed more of a fibrous structure. The surface of both bilayer membranes was covered with a more compact layer. In vivo, both membranes were integrated into the surrounding tissue without any inflammatory reaction. Additionally, a considerable biodegradation was noted within four-to-eight weeks with BG, while the resorption of RM primarily occurred within the first eight-to-twelve weeks. Within the limits of this present study, it can be concluded that all examined membranes show a high degree of biocompatibility. RM and BG are resorbed without inflammation within 8 (BG) or 12 weeks (RM).[23]

Porcine pericardium has found its applications in the field of implantology. A case report describes the treatment of horizontal osseous defects around implants in a one-stage split-mouth approach in a middle-aged female patient. Different biomaterials: a-tricalciumphosphate (Ceros TCP granules) grafting material and a porcine, collagen pericardium resorbable membrane (Remotis) were employed on the test side and a deproteinized, bovine bone matrix (Bio-Oss) and a porcine, collagen resorbable membrane (Bio-Gide) on the control side. Bone substitutes were mixed with autologous bone (approximately $10 \%$ ) harvested during implant insertion procedures. Substantial bone regrowth was evident in both ridges, although only the test side underwent complete regeneration. Histological examination of the regenerated areas showed the presence of mature bone organized around particles of biomaterial during the incorporation phase.[24] An experimental study investigated the Bone Morphometric evaluation around immediately placed Implants covered with Porcine-Derived Pericardium Membrane. Twenty-four commercially available endosseous implants were placed in the fresh extraction sockets of the mandibular first molar of mature beagles. On one side, implants and osteotomy sites were covered with porcine-derived bioresorbable pericardium membranes, whereas on the other side, no membranes were used. After 6 weeks, samples were retrieved and were histologically processed for histomorphometric analysis. The histological observation showed that bone loss and soft tissue migration in the 
coronal region of the implant were evident for the control group, whereas bone fill was evident up to the neck of the implant for the membrane-covered group. Bone-to-implant contact was significantly higher for the membrane-covered group compared to the control group, $75 \%$ and $45 \%$, respectively. The experimental membranes proved to regenerate bone around implants placed in fresh extraction sockets without soft tissue intrusion. [25]

\section{Human Pericardium}

The advantages of pericardium membranes are manifold and hence apart from bovine and porcine pericardium, human pericardium membranes have been developed. Bovine and HP membranes were evaluated in a study by Thomaidis et al. Fifty adult male New Zealand white rabbits were used in this study. Five groups of 10 animals each were used: Human fascia lata membrane, HP, human fascia temporalis, bovine pericardium (BP), and expanded polytetrafluoroethylene (e-PTFE). According to the results, the fascia lata, HP, BP, and ePTFE advance bone regeneration and can be successfully used as GBR membranes for osseous defects beyond the critical size. The defect in the HP group was filled with a mature, mainly newly formed bone, lamellar in its greatest part, with distinct bone trabeculae and marrow sites. [26] The biochemical and biomechanical evaluation of new bone formed in bone defects was assessed by biomechanical hardness tests and alkaline phosphatase activity after a period of 60 days. The experiment was performed by creating defects $8 \mathrm{~mm}$ in diameter in human calvarium, by using demineralized bone matrix as control. The results concluded that the human pericardium had higher biomechanical hardness. Hence human pericardium can be considered as a suitable bone graft in bone repair leading to acceptable bone strength.[27] Human pericardium membrane has been tested for its feasibility to augment localized alveolar ridge defects for the subsequent placement of dental implants in incompletely healed extraction sockets. Horizontal (width) and vertical hard tissue measurements with CBCT were recorded on the day of ridge augmentation surgery, 4 month and 7 months follow-up. Bone volume achieved through guided bone regeneration was a gain of $4.8 \mathrm{~mm}$ horizontally (width) and $6.8 \mathrm{~mm}$ vertically in the deficient ridge within a period of 7 months following the procedure. The results suggested that HP Allograft membrane may be a suitable component for augmentation of localized alveolar ridge defects.[28]A combination of decalcified freeze-dried bone allograft and resorbable human pericardium, in conjunction with cortical channel expansion, was used for the guided bone augmentation for treatment of a facial maxillary alveolar bone defect to enhance the esthetic result for an all ceramic fixed partial denture (FPD). Post-operative examinations showed improvement in the alveolar bone contour. The regeneration of the missing osseous structure was accomplished to support the future esthetic soft tissue contours. This osseous regenerative technique significantly increased the functional and esthetic outcome of the final FPD by restoring the alveolar ridge defect to its original dimension. [29]

\section{Conclusion}

The criteria required to select appropriate barrier membranes for guided bone regeneration encompass biocompatibility, integration by the host tissue, cell occlusiveness, space-making ability and adequate clinical manageability. The difficulty of maintaining the barrier function for an appropriate length of time is considered a major drawback of resorbable membranes. Although collagen membranes show an effective regeneration profile, however their major drawback is their fast resorption, resulting in early loss of barrier function. The pericardium membranes, in comparison to collagen membranes have shown an effective crosslinking, suggesting prolonged resorption time. Various in vitro and in vivo studies have managed to prove the bioeffectiveness of these pericardium membranes in enhancing bone augmentation. However, more clinical trials are required to prove their efficiency in guided bone regeneration.

\section{Acknowledgements}

Author would like to thank Dr. Sanjana Ravi Kumar (MDS, Endodontics) and Dr. Sumeet Gupta( BDS) for their help and contribution in data collection and manuscript editing.

\section{References}

[1]. Nyman. S, Lindhe. J, Karring. T and Rylander. H, New attachment following surgical treatment of human periodontal disease, J Clin Periodontol,9, 1982, 290-296.

[2]. Inoue. H, Igur.o Y, Matsumoto. H, Ueno. M, Higashi .A, Sakata .R, Right hemi-reconstruction of the left atrium using two equine pericardial patches for recurrent malignant fibrous histiocytoma: report of a case, Surg Today, 39(8), 2009, 710-712.

[3]. Shinn. SH, Sung .K, Park. PW, Lee. Y.T, Kim. W.S, Yang. J.H et al. Results of annular reconstruction with a pericardial patch in active infective endoarditis. J Heart Valve Dis, 18(3),2009,315-320.

[4]. ASM International. Materials and Coatings for Medical Devices: Cardiovascular. ASM International; OH, USA: 2009.

[5]. Li. X, Guo. Y, Ziegler. Model. L, Eghbalieh .S DD, Brenes. R K et al. Current usage and future directions for the bovine pericardial patch, Ann Vasc Surg, 25(4), 2011, 561-568.

[6]. Muto .A, Nishibe .T, Dardik. H, Patches for carotid artery endarterectomy: Current materials and prospects, J Vasc Surg,50(1),2009,206-213. 
[7]. Goissis. G, Giglioti .A F, Braile. DM. Preparation and characterization of an acellular bovine pericardium intended for manufacture of valve bioprostheses, Artif Organs,35(5),2011,484-489.

[8]. Guldner NW, Bastian F, Weigel G, et al. Nanocoating with titanium reduces iC3b- and granulocyte-activating immune response against glutaraldehyde-fixed bovine pericardium: a new technique to improve biologic heart valve prosthesis durability?, J Thorac Cardiovasc Surg, 143(5),2012,1152-1159.

[9]. Mathapati S, Bishi DK, Venugopal J R, Cherian K M, GuhathakurtaS, RamakrishnaS, VermaS R, Nanofibers coated on acellular tissue-engineered bovine pericardium supports differentiation of mesenchymal stem cells into endothelial cells for tissue engineering, Nanomedicine, 9(5), 2012,623-634.

[10]. Zhang W, Wu H, Yao B, Hu G, Zhou Y, Experimental study on acellular bovine pericardium guided bone regeneration material, Zhongguo Xiu Fu Chong Jian Wai Ke Za Zhi.,20(3),2006,287-291.

[11]. Chang. Y, Lai .PH, Wei. HJ , W.W.Chen, C.H.Hwang, S.M, et al. Tissue regeneration observed in a basic fibroblast growth factorloaded porous acellular bovine pericardium populated with mesenchymal stem cells, J Thorac Cardiovasc Surg.134(1),2007,65-73, 73.e1-4

[12]. Steigmann M, Pericardium membrane and xenograft particulate grafting materials for horizontal alveolar ridge defects. Implant Dent, 15,2006, 186-91

[13]. Sterio. TW, Katancik. JA, Blanchard. SB, Xenoudi .P, Mealey .BL. A prospective, multicenter study of bovine pericardium membrane with cancellous particulate allograft for localized alveolar ridge augmentation. Int J Periodontics Restorative Dent.33(4),2013,499-507.

[14]. Bai M, Zhang T, Ling T et al. Guided bone regeneration using acellular bovine pericardium in a rabbit mandibular model: in-vitro and in-vivo studies. J Periodontal Res. 49(4),2014, 499-507.

[15]. Zhou YQ, Wu HJ. Experimental study on guiding bone regeneration with bovine pericardium membrane. Hua Xi Kou Qiang Yi Xue Za Zhi. 22(5),2004, 429-431.

[16]. Ahn. YS, Kim. SG, Kim. CS, Oh. JS, Lim .SC . Effect of guided bone regeneration with or without pericardium bioabsorbable membrane on bone formation. Oral Surg Oral Med Oral Pathol Oral Radiol.,114(5 Suppl): S 2012,126-31.

[17]. Wu. H, Hu. G. Comparison of acellular bovine pericardium material with collagen membrane in guiding bone regeneration. Zhongguo Xiu Fu Chong Jian Wai Ke Za Zhi.21(7),2007,743-747.

[18]. Stavropoulos. A, Chiantella .G, Costa .D, Steigmann.M, Windisch. P, Sculean. A . Clinical and Histologic Evaluation of a Granular Bovine Bone Biomaterial Used as an Adjunct to GTR With a Bioresorbable Bovine Pericardium Collagen Membrane in the Treatment of Intrabony Defects. J Periodontol, 82,2011, 462-470.

[19]. Schlee.M, Ghanaati. S, Willershausen. I, Stimmlmayr. M, Sculean .A, Sade .R A. Bovine pericardium based non-cross linked collagen matrix for successful root coverage, a clinical study in human. Head \& Face Medicine, 8(6),2012.

[20]. Fu. JH, Oh .TJ, Benavides. E, Rudek. I, Wang .HL. A randomized clinical trial evaluating the efficacy of the sandwich bone augmentation technique in increasing buccal bone thickness during implant placement surgery I. Clinical and radiographic parameters. Clinical Oral Implants Research,25(4), 2014,458-467.

[21]. Lam MT, Wu J C .Biomaterial applications in cardiovascular tissue repair and regeneration. Expert Rev Cardiovasc Ther. 2012;10(8): 1039-1049.

[22]. Morticelli. L, Thomas. D, Roberts. N, Ingham. E, Korossis. S. Investigation of the suitability of decellularized porcine pericardium in mitral valve reconstruction, J Heart Valve Dis,22(3),2013, 340-353.

[23]. Rothamel. D, Schwarz. F, Fienitz. T, Smeets ..R, Dreiseidler T, Ritter. L et al. Biocompatibility and biodegradation of a native porcine pericardium membrane: results of in vitro and in vivo examinations.Int J Oral Maxillofac Implants,27(1),2012,146-154

[24]. Merli.M,Moscatelli.M,Mazzoni.A,Merli.M,Mariotti.G, Nieri.M,Lateral bone augmentation applying different biomaterials. A clinical and histological evaluation of a case report, Z Zahnarztl Implantol, 29, 2013,70-79.

[25]. Jimbo R,Marin C,Witek L,Suzuki M, Tovar N, MateiI C, Dragan I F, Coelho P G. Bone Morphometric Evaluation around Immediately Placed Implants Covered with Porcine-Derived Pericardium Membrane: An Experimental Study in Dogs. International Journal of Biomaterials ,2012, 7 pages

[26]. Thomaidis V, Kazakos K, Lyras DN, Dimitrakopoulos I, Lazaridis N, Karakasis D, et al. Comparative study of 5 different membranes for guided bone regeneration of rabbit mandibular defects beyond critical size. Med Sci Monit ,14,2008, 67-73.

[27]. Alireza Nasoori, Soroush Mohitmafi, Ahad Khoshzaban,Seyed Amir Hosein Tavakoli,Zohreh Shahabi. Biochemical and biomechanical evaluation of human pericardial membrane and demineralized bone matrix in rabbit calvarial defects, Comparative Clinical Pathology ,22(2),2013, 253-259.

[28]. Vidyadharan .AK, Ravindran. A. Localized ridge defect augmentation using human pericardium membrane and demineralized bone matrix, Indian J Dent Res,25, 2014,445-448.

[29]. Taskonak .B, Ozkan. Y. An alveolar bone augmentation technique to improve esthetics in anterior ceramic FPDs: a clinical report, J Prosthodont, 15,2006,32-36. 